\title{
Indium Tin Oxide Microsystem for Electrochemical Detection of Exocytosis of Migratory Dendritic Cells
}

\author{
Xiaoqing Liu, ${ }^{a, b}$ Marine Bretou, ${ }^{c}$ Ana-Maria Lennon-Duménil, ${ }^{c}$ Frédéric Lemaître, ${ }^{a, b}$ Manon Guille-Collignon ${ }^{a, b^{*}}$
}

a Ecole normale supérieure, PSL Research University, UPMC Univ Paris 06, CNRS, Département de Chimie, PASTEUR, 24, rue Lhomond, 75005 Paris, France

Sorbonne Universités, UPMC Univ Paris 06, ENS, CNRS, PASTEUR, 75005 Paris, France

c INSERM U932, Inst Curie, 12, rue Lhomond, 75005 Paris, France

* e-mail: manon.guille@ens.fr

\begin{abstract}
The design, fabrication and test of an indium tin oxide (ITO) microdevice to investigate exocytotic behaviors of migratory dendritic cells (DCs) in confined three-dimensional environment were reported in this work. Indeed, immature DCs were able to migrate into micro-fabricated biocompatible polydimethylsiloxane (PDMS) channels that mimic their natural constrained environment of tissues for patrolling in search of danger associated antigens through an endocytotic process called macropinocytosis. In order to coordinate membrane trafficking and prevent cell volume increment, DCs will release part of their contents back to the extracellular medium while migrating. Through electrochemical measurements, we demonstrated that exocytotic events of migratory DCs could be monitored by our ITO microdevice. In addition, the transparency of ITO electrode should facilitate future combining assays of exocytosis with other fluorescence-based measurements of cell physiology.
\end{abstract}

Keywords: Electrochemical detection; Exocytosis; ITO microsystem; Dendritic cells; Cell migration

Dendritic cells (DCs), the most potent antigen (AG) presenting cells, are the sentinels of the immune system. ${ }^{[1-3]}$ They effectively patrol peripheral tissues in search of danger associated AG through a biological phenomenon called "macropinocytosis". This process constitutes the first step of the adaptive immune response and significantly relies on specific DCs properties, including their high endocytic capacity ${ }^{[4,5]}$ as well as their efficient motility ${ }^{[6]}$ in confined three-dimensional (3D) environments. Macropinocytosis represents a distinct pathway of endocytosis in mammalian cells (Scheme 1). ${ }^{[7-9]}$ It includes the uptake of cargo from the extracellular medium by macropinosomes, the preservation of the surface membrane area, and the regulation of membrane traffic along the intracellular pathway. Interestingly, previous study [7] shows that macropinosome traffic is not unidirectional (endocytic), but bidirectional (endocytic and exocytotic), indicating that DCs macropinocytosis is accompanied by exocytotic events. Recently, high resolution time-lapse imaging confirms that exocytosis is taking place when DCs migrate in the confined 3D microchannels. ${ }^{[10]}$ Such secretory events might favor matrix remodeling and membrane recycling required for cell migration. Moreover, this exocytotic process taking place in DCs plays an important role in proper cell communication/activation, and need to be tightly controlled in order to allow either an efficient clearance of pathogens, or the maintenance of immune homeostasis. Characterization of exocytosis of migratory DCs is thus significant to better understand the cellular trafficking in 3D-confinement. In this context, secretion coupled to cell 
migration has been poorly addressed. This is why we considered to use amperometry in 3D-confined microchannels that mimics the confined environment in which DCs migrate. Indeed, amperometry is a routine analytical technique for quantitative, real-time monitoring of exocytosis at single cell level. ${ }^{[1-13]}$ In general, a microelectrode is positioned in the close vicinity of a target cell during amperometric measurement, either on the top or at the bottom, and a constant potential is applied to the working electrode at which electrochemical biomolecules (mainly catecholamines) released during exocytosis could be oxidized. As a consequence, the exocytotic fluxes are depicted as a series of amperometric current spikes and each single spike results from an individual exocytotic event. ${ }^{[14]}$ The frequency and shapes of amperometric spikes elucidate particular information about the dynamics of the release process while their integrals give the exact quantity of electroactive molecules released. Different microelectrodes (carbon fiber $\left.{ }^{[11,14-16]}, \mathrm{Au}{ }^{[17]}, \mathrm{Pt}{ }^{[18]} . ..\right)$ have been employed for amperometric detection of electroactive molecules discharged from individual vesicle during exocytosis. Among them, indium tin oxide (ITO) microelectrodes appear to be a good choice due to their excellent optical transparency, high electrical conductivity as well as their appropriate electrochemical working window for the electroanalysis of biomessengers. Our group and others have been developing microchips based on transparent ITO microelectrodes on a glass substrate in order to facilitate higher throughput measurements of exocytosis as well as to enable fluorescent measurements that are not possible with usual carbon-fiber electrodes. ${ }^{[19-23]}$

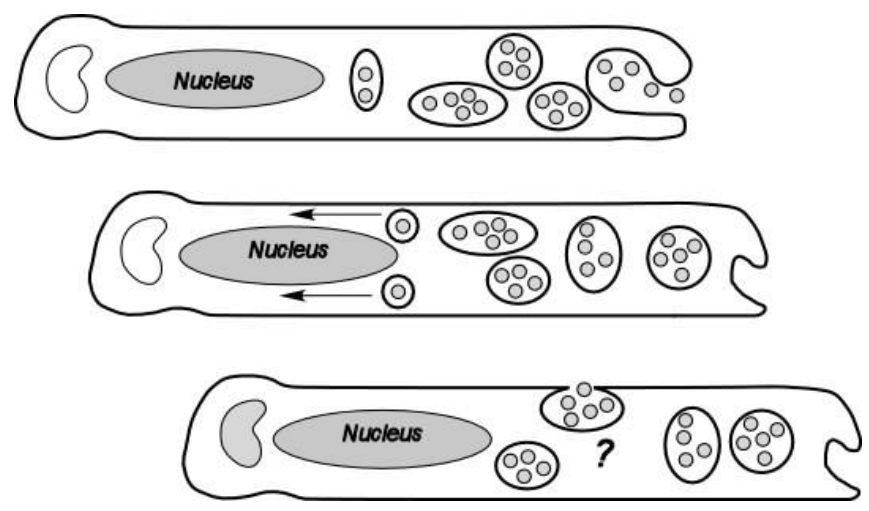

Scheme 1. Schematic view of macropinocytosis process. Top : Formation of macropinosomes at the front of the migrating dendritic cell while it is moving. Middle : Antigen presentation towards the back of the cell, behind the nucleus. Vesicles undergo series of morphological changes to concentrate the internalized antigen. Bottom: Possible exocytotic event towards the extracellular medium so as to achieve the membrane retrieval and excessive fluid discharge.
To the best of our knowledge, there have been so far few researches focusing on studies of exocytotic behaviors of DCs. In 2006, by means of fluorescent microscopy, macropinocytosis of DCs was found to be a regulated coordination of endocytic and exocytotic membrane traffic events. ${ }^{[7]}$ The membrane release of serotonin from DCs upon ATP stimulation was also confirmed by amperometric measurement with carbon fiber microelectrode. ${ }^{[24]}$ However, these studies have been mainly performed ex vivo on flat two-dimensional (2D) surfaces and such substrates rarely apply to in vivo cell migration, which mainly occurs in $3 \mathrm{D}$ confined environment. Exocytotic behaviors of migratory DCs in $3 \mathrm{D}$ confinement, as in vivo, were investigated in this article by designing and fabrication of a PDMS microchannels/ITO microsystem (Fig.1 (A)) allowing simultaneous observation of cells and amperometric detection of exocytosis as described herein. (The details concerning ITO microdevice assembly are shown as supporting information in Fig.S-1)
(A)

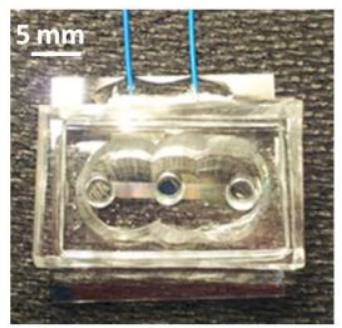

(C)

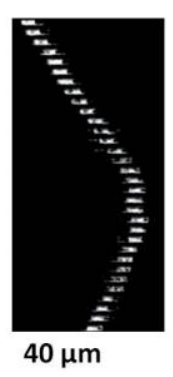

(D)

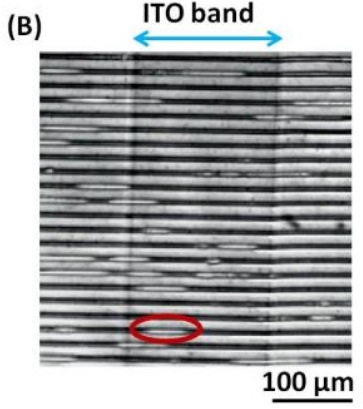

$100 \mu \mathrm{m}$

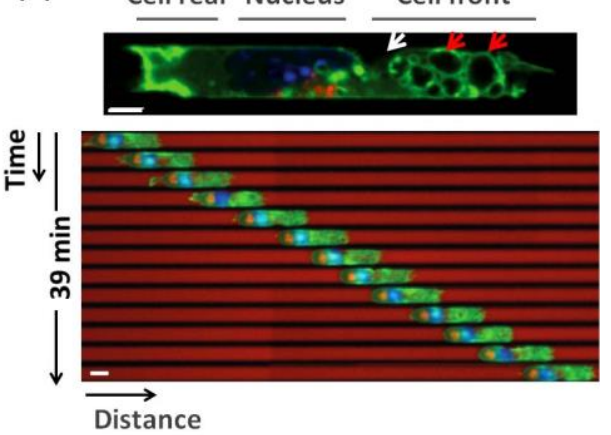

Figure 1. (A) ITO microdevice for amperometric detection; (B) DCs migrating to the fibronectin coated ITO/glass substrate; the blue double headed arrow depicts the position of the transparent ITO microelectrode and the red ellipse indicates the single migratory DC lying on ITO band; (C) A typical example of an individual DC migrating in $4-\mu \mathrm{m}$ width*5- $\mu \mathrm{m}$ height fibronectin-coated microchannels. Sequential images were processed to remove the channels so that only the cells are visible (white). Temporal sequences were put one below the other (Magnification $10 \times$, the interval between two images is 2 min, scale bar $40 \mu \mathrm{m}$ ). (D) Top: A representative Life-act-GFP $\mathrm{DC}$ in $8-\mu \mathrm{m}$ width $* 5-\mu \mathrm{m}$ height fibronectin-coated 
microchannels: the nucleus was stained with Hoechst (in blue); lysosomes in red were tagged with WGA-AF647 and macropinosomes of the migrating DC were indicated by the white arrows at the cell front (Magnification 60x); Sequential images of the life-act-GFP DC migrating in microchannels filled with OVA AF-647 (in red). Temporal sequences were put one below the other (Magnification 20x, one image every 3 min, scale bar: $5 \mu \mathrm{m}$ ). DC was able to internalize the OVA-647 in the channels and it was finally accumulated into the lysosomes at the cell rear during migration Several events of secretions might be envisionned for a single DC. ${ }^{[24]}$ For instance, a vesicle formed at the cell front could be exocytosed (indicated by the red arrow in the top image).

3D-confined microchannels made of PDMS, a biocompatible silicone elastomer, have been demonstrated to be able to induce DCs to move inside the microchannels at speeds similar to those observed in vivo. ${ }^{[6,10,25,26]}$ In our case, the transparent property of the bottom ITO-embedded glass slide allowed us to observe DCs' behaviors through high-resolution microscopy. Additionally, multiple parallel channels in each device (around 80 channels per chamber), enabled an easy recording of many cells' behaviors at low magnification. As shown in Fig.1 (B), after overnight incubation, DCs previously loaded into the entry plug of the ITO microdevice spontaneously entered into PDMS microchannels and were able to move across the ITO electrode, without any mechanical or chemical stimulation. By tracking DCs' migration along PDMS microchannels with time-lapse imaging, we noticed that not all the cells acted at the same way inside the microchannels (see movie 1 shown in supporting information). Firstly, there were big differences of cell sizes; secondly, most cells underwent important speed fluctuations during motion, resulting in different migration distances; finally, some cells showed changes in direction during migration (Fig.1 (C)). However, after overnight incubation, we always saw DCs moving on ITO electrode.
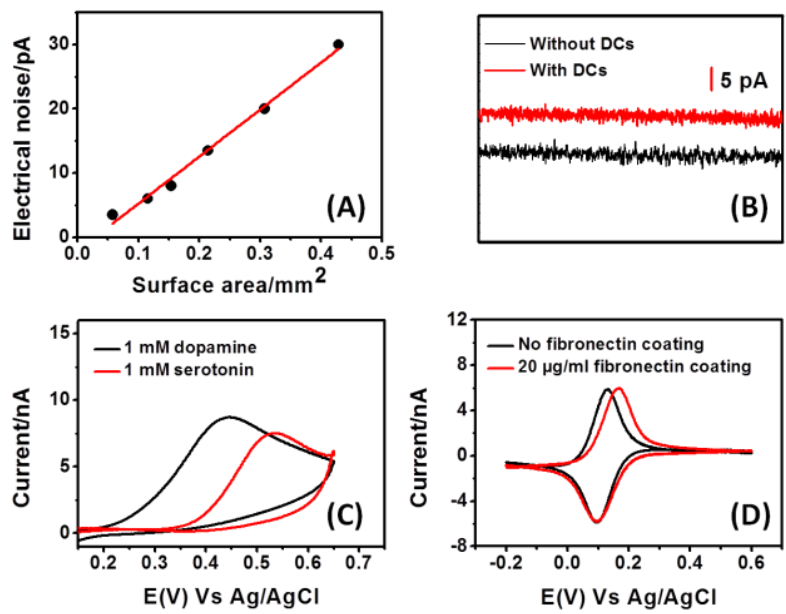

Figure 2. (A) Measurements of the electrical noise in PBS $(\mathrm{pH}=7.4)$ at a constant potential value $(\mathrm{E}=+650 \mathrm{mV}$ vs. $\mathrm{Ag} / \mathrm{AgCl}$ ) as a function of electrodes dimensions ( $\mathrm{y}=70 \mathrm{x}-2$, $\mathrm{R}^{2}=0.948$ ); (B) Electrical noise of ITO microelectrode with and without DCs and its dimension is of $200 \mu \mathrm{m}$ by $320 \mu \mathrm{m} ;(\mathrm{C})$ Cyclic voltammograms of $1 \mathrm{mM}$ serotonin and $1 \mathrm{mM}$ dopamine in PBS solution $(\mathrm{pH}=7.4)$ respectively, scan rate $20 \mathrm{mV} / \mathrm{s}$. (D) Cyclic voltammograms of $100 \mu \mathrm{M} \mathrm{K} \mathrm{K}_{3} \mathrm{Fe}(\mathrm{CN})_{6}$ in PBS $(\mathrm{pH}=7.4)$ on ITO electrode with and without fibronectin treatment, scan rate $20 \mathrm{mV} / \mathrm{s}$.

As shown in Fig.1(D), by staining the different compartments of DCs with specific dyes, we found that while migrating along the microchannels, DCs could form lots of macropinosomes (as indicated by the white arrows in Fig.1(D)) in front of the nucleus to sample the microenvironment so as to take up extracellular antigens and the internalized antigens were accumulated in lysosomes at the cell rear (Fig.1(D)). We thus believe that migrating DCs are able to expel part of their contents to the extracellular space so as to achieve the coordination of endocytic and exocytotic membrane trafficking. That is, exocytotic events are properly taking place during migration. Previous studies have shown that DCs are able to not only accumulate neurotransmitters but also synthesize, store and degrade them. Since neurotransmitters play important roles in immune response of $\left.\mathrm{DCs},{ }^{[27,} 28\right]$ we try to figure out if there are electroactive neurotransmitters, like serotonin or dopamine, expelled by DCs during migration with amperometric detection. ${ }^{[24,29-31]}$ This is different from what have been shown before in the literature in the way that secretion is monitored here while the DCs are confined in 3D microdevices and are migrating into the ITO microsystem channels assembly.

Fig.2 (A) presents the relationship between the current noise and the surface area of ITO working electrode of which the band widths varied from $200 \mu \mathrm{m}$ to $1000 \mu \mathrm{m}$. Globally the line depicts a good linear fit with a slope of $0.07 \mathrm{fA} / \mu \mathrm{m}^{2}$. Moreover, the best electrical properties were obtained from $200 \mu \mathrm{m}$ ITO band with minimum surface area of $0.06 \mathrm{~mm}^{2}$, with a rms noise of typically 2 to $4 \mathrm{pA}$, which is consistent with our previous work based on ITO microelectrodes. ${ }^{[22]}$ We also compared the current noises obtained from ITO microelectrodes with and without DCs. As illustrated in Fig.2 (B), migratory DCs adjacent to ITO electrodes didn't increase the background noise for amperometric recordings. All measurements were performed in PBS (phosphate buffer saline) solution and no obvious difference was seen for the two parallel ITO microelectrodes in the same microdevice.

Fig.2 (C) shows cyclic voltammograms of two wellknown neurotransmitters $(1 \mathrm{mM}$ serotonin and $1 \mathrm{mM}$ dopamine) inside the 3D-confined microchannels 
collected on ITO microelectrode at a scan rate of $20 \mathrm{mV} / \mathrm{s}$. We can see that dopamine started to be oxidized at about $+200 \mathrm{mV}$ while the oxidation of serotonin occurred at $+400 \mathrm{mV}$, and their oxidation peaks were located at around $+450 \mathrm{mV}$ and $+550 \mathrm{mV}$, respectively. According to the results mentioned above, our ITO microdevice is adequate from an electrochemical point of view for amperometric detection of these electroactive neurotransmitters release. Therefore, in the following experiments, we applied a potential of $+650 \mathrm{mV}$ to track neurotransmitter release from DCs on ITO electrode by amperometric measurement, this value being identical with the one we used to detect serotonin discharged from cells by carbon-fiber and ITO electrodes in previous references. ${ }^{[22,23,32]}$

In our microdevice, the bottom surface for DCs' migration is composed by both ITO and glass, showing inhomogeneity, further surface treatment is thus necessary to let DCs adhere and spread in PDMS microchannels. For the sake of creating a homogenization of the whole surface, we pre-coated the microchannels with $20 \mu \mathrm{g} / \mathrm{mL}$ fibronectin solution for one hour. To investigate whether electrochemical properties of ITO electrodes were affected by fibronectin treatment, we compared cyclic voltammogram of a well characterized redox mediator $\mathrm{K}_{3} \mathrm{Fe}(\mathrm{CN})_{6}(100 \mu \mathrm{M})$ acquired on naked ITO microelectrode with that obtained from $20 \mu \mathrm{g} / \mathrm{mL}-$ fibronectin precoated ITO band. As shown in Fig.2 (B), besides the slightly shift of the oxidation peak, fibronectin coating did not alter the electrochemical detection at ITO microelectrodes.

In our 3D-confined PDMS microchannels that mimic the natural constrained environment of tissues, DCs were able to migrate through the ITO microelectrode (Fig.1(B)). Previous study shows that for migrating DCs, exocytosis was taking place so as to empty part of the ingested fluid as well as to recycle the internalized membrane during macropinocytosis (Fig.1(D)). ${ }^{[10]}$ In this present work, based on ITO microelectrode, we tried to investigate the spontaneous exocytotic events of migratory DCs by amperometric detection, which provides the accurate amount of released biomolecules and precise kinetic characteristics with high sensitivity and sub-millisecond temporal resolution.

We applied a $+650 \mathrm{mV}$ constant potential at the ITO electrode vs. $\mathrm{Ag} / \mathrm{AgCl}$ and recorded the current as a function of time. The exocytotic release trace from migratory DCs in 3D-confined PDMS microchannels thus appeared as a succession of individual, well separated amperometric spikes, as presented in Fig.3 (A).
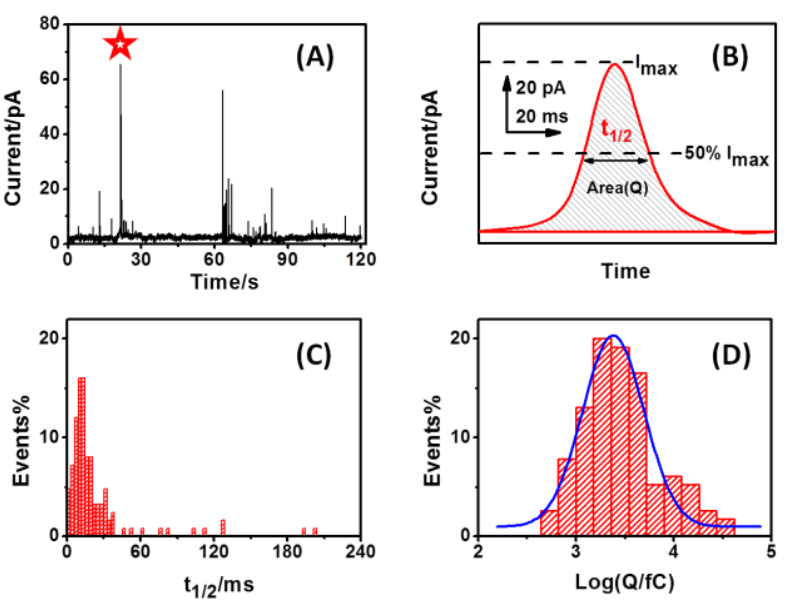

Figure 3. (A) Representative amperometric trace monitored on the 3D-confined ITO microdevice from migratory DCs; (B) A typical amperometric spike extracted from amperometric trace (indicated by the red star); (C) Histogram of half-height width $\left(\mathrm{t}_{1 / 2}\right)$; (D) Statistical logarithmic distributions ( $\mathrm{y}=$ $\left.0.2 \mathrm{e}^{-5.6(\mathrm{x}-3.4)^{2}}, \mathrm{R}^{2}=0.932\right)$ of the charge $\mathrm{Q}$ obtained for each exocytotic event electrochemically detected on the 3D-confined ITO microdevice for DCs.

At a potential at the foot of the oxidation waves of dopamine or serotonin (see Fig.2 (C), respectively, 100 and $300 \mathrm{mV}$ vs. $\mathrm{Ag} / \mathrm{AgCl}$, no spikes were detected (data not shown). The current spikes suggested electroactive biomolecules were discharged by migratory DCs and then immediately oxidized at ITO surface, confirming the viewpoint that macropinocytosis of migratory DCs is accompanied by exocytotic events, resulting in the coordination of endocytic and exocytotic membrane trafficking. It is important to note that this secretion is detected in the absence of any stimulation/secretagogue nor prior loading of the cells with any neurotransmitter or electroactive molecule, pointing out the facts that the DCs have endogenous electroactive neurotransmitters ${ }^{[28]}$ and that the spontaneous secretion is probably triggered by confinement. Indeed the same experiments performed in Petri dishes with the technique of carbon fiber microelectrodes used by amperometry showed no secretion in the absence of stimulation or prior loading of the cells (data not shown).

Fig.3(B) displays a typical single oxidative spike on expanded time scale extracted from Fig.3(A), which is corresponding to an individual exocytotic event. In amperometric measurement, maximum current amplitude $\mathrm{I}_{\max }(\mathrm{pA})$, half-height width $\mathrm{t}_{1 / 2}(\mathrm{~ms})$ and area $\mathrm{Q}(\mathrm{pC})$ are typical characteristics for a single spike analysis. Note that $I_{\max }$ represents the maximum release flux, $t_{1 / 2}$ is defined as the time interval where current amplitude exceeds $50 \%$ of $\mathrm{I}_{\max }$ while the integral charge $\mathrm{Q}$ was employed to calculate the precise release amount on the 
base of Faraday's law. For migratory DCs, we analyzed the results from a total of 125 events with a wide range of amplitudes varying from 5 to $120 \mathrm{pA}$ (mean value, 21.9 $\pm 3.4 \mathrm{pA}$ ) and histograms of $\mathrm{t}_{1 / 2}$ and integral $\mathrm{Q}$ were presented separately in Fig.3(C) and Fig.3(D). The distribution of $t_{1 / 2}$ values shows a half-height width of a few milliseconds, with a mean value of $25.05 \pm 3.44 \mathrm{~ms}$ and the mean $\mathrm{Q}$ value is $0.74 \pm 0.16 \mathrm{pC}$. However, larger, more irregularly shaped events also occured (see Fig.S-2 shown in supporting information). These spikes may come from sites further from ITO microelectrode or represent multiple releases or another type/size of vesicles.

We have designed and manufactured an effective 3Dconfined ITO/PDMS microdevice which allows simultaneously cell observation and amperometric measurement of exocytosis from migratory DCs. The phenomenon that macropinocytosis of migratory DCs is accompanied by exocytotic events was monitored by amperometric detection. We propose that our experiments will identify specific modes of exocytosis and will display the mutual interaction of endocytic and exocytotic trafficking of DCs as well as will quantitatively analyzing exocytosis during cell migration. In addition, further optimization of our microdevice based on transparent ITO microelectrode should facilitate future real-time combining assays of DCs' exocytotic behaviors with other fluorescence technology. ${ }^{[23,33,34]} \mathrm{By}$ doing so, correlations between place (on the DC surface, rear or front) or size of vesicles (lysosomes, macropinosomes etc...) and quantitative parameters of secretion could be drawn.

\section{Experimental}

ITO microelectrodes were manufactured by photolithographic process and acidic wet etching (see Fig.S-1(A) shown in supporting information). A thin film of ITO $\left(90 \% \mathrm{In}_{2} \mathrm{O}_{3} / 10 \% \mathrm{SnO}_{2} ; 150 \mathrm{~nm}\right.$ thickness; ACM, Villiers Saint Frédéric, France) was previously sputtered onto optical glass slides $(22 \mathrm{~mm} \times 22 \mathrm{~mm} \times 0.13 \mathrm{~mm})$ in order to afford a material of low electrical resistance $(\leq$ 20 ohms per square) and transparency. To be specific, firstly, an insulating photoresist (AZ 9260, Clariant $\mathrm{GmbH}$, Germany) was patterned onto the ITO/glass slides by the following steps: (a) spin-coating (b), prebaking on a hotplate at $110^{\circ} \mathrm{C}$, exposure to UV light through a specific mask design (c) and development (AZ $400 \mathrm{~K}+\mathrm{H}_{2} \mathrm{O}$ ) (d). Then, the ITO with no "photoresist protective layer" was chemically etched by commercial $\mathrm{HCl}$ solution and the photoresist remaining on ITO band was removed with acetone. This 'wet etching' step left two identical ITO microelectrodes of about $200-\mu \mathrm{m}$ width respectively on the glass slide.

Biocompatible PDMS microchannels with the inlet and outlet ports were prepared as previously described. [25, 26] In brief, microchannels were fabricated in PDMS using rapid prototyping and soft lithography. As a result, for each PDMS chamber (see Fig.S-1(B) shown in supporting information), multiple microchannels are run in parallel which makes it suitable for multiple cells observation. Moreover, we fixed a homemade PDMS gasket with diameter of $1.5 \mathrm{~cm}$ onto the top of the PDMS microchannels so as to hold the culture medium, making the chamber an appropriate environment for cells ((see Fig.S-1(C) shown in supporting information)). PDMS chamber and the ITO-embedded glass slide were then activated in a plasma cleaner (PDC-32G Harrick) for 1 min at 400 mTorr and were stuck together. One hour incubation in $60{ }^{\circ} \mathrm{C}$ oven was used to strengthen the binding. The upper surface of the microchip was then activated by plasma treatment for $2 \mathrm{~min}$ at $400 \mathrm{mTorr}$ to produce hydrophilic inlet which enabled $20 \mu \mathrm{g} / \mathrm{mL}$ fibronectin (fibronectin from bovine plasma $0.1 \%$ solution) to fill into the microchannels. After one hour coating at room temperature, the microchip was rinsed 3 times with PBS. The system could be used immediately or stored up to 3 days at $+4{ }^{\circ} \mathrm{C}$. Before loading cells, microchannels were washed twice with cell culture medium and kept in the incubator while collecting cells.

Amperometric detection of exocytosis at ITO microelectrodes was carried out using a picoamperometer (model AMU-130, Radiometer Analytical Instruments, Copenhagen, DK) at a constant potential $\mathrm{E}=+650 \mathrm{mV}$ vs. a silver/silver chloride reference electrode $(\mathrm{Ag} / \mathrm{AgCl}$, a 1-mm diameter wire). The time course of the amperometric current was monitored (output digitized at $40 \mathrm{kHz}$ ) and stored on a computer (Latitude D600, Dell) through a D/A converter (Powerlab 4SP, AD Instruments) and its software interface (Chart version 4.2 for Windows, ADInstruments). The significative parameters of an amperometric spike are the maximum oxidation current $\mathrm{I}_{\max }(\mathrm{pA})$, the half-height width $t_{1 / 2}(m s)$ and the total electrical charge $Q(p C)$. All the values are presented as the mean \pm s.e.m. (standard error of the mean $=$ standard deviation $/ \sqrt{n}$, where $\mathrm{n}$ is the number of spikes analyzed). Voltammetric analyses were performed with an EA162 Picostat (eDAQ, Australia) through an e-corder 401 system associated with EChem software. The reference electrode was also $\mathrm{Ag} / \mathrm{AgCl}$. 


\section{Acknowledgements ((optional))}

This work has been supported in part by CNRS (UMR 8640), Ecole Normale Supérieure (PSL Research University), French Ministry of Research and Université Pierre \& Marie Curie Paris 6 (Sorbonne Universités). M.

\section{References}

[1] M. F. Lipscomb, B. J. Masten, Physiol. Rev. 2002, 82, 97-130.

[2] J. Banchereau, R. M. Steinman, Nature 1998, 392, 245252.

[3] J. Banchereau, F. Briere, C. Caux, J. Davoust, S. Lebecque, Y. T. Liu, B. Pulendran, K. Palucka, Annu. Rev. Immunol. 2000, 18, 767-+.

[4] A. E. Morelli, A. T. Larregina, W. J. Shufesky, M. L. G. Sullivan, D. B. Stolz, G. D. Papworth, A. F. Zahorchak, A. J. Logar, Z. L. Wang, S. C. Watkins, L. D. Falo, A. W. Thomson, Blood 2004, 104, 3257-3266.

[5] W. S. Garrett, L. M. Chen, R. Kroschewski, M. Ebersold, S. Turley, S. Trombetta, J. E. Galan, I. Mellman, Cell 2000, 102, 325-334.

[6] M. L. Heuze, P. Vargas, M. Chabaud, M. Le Berre, Y.-J. Liu, O. Collin, P. Solanes, R. Voituriez, M. Piel, A.-M. Lennon-Dumenil, Immunol. Rev. 2013, 256, 240-254.

[7] S. Falcone, E. Cocucci, P. Podini, T. Kirchhausen, E. Clementi, J. Meldolesi, Journal of Cell Science 2006, 119, 4758-4769.

[8] A. T. Jones, Journal of Cellular and Molecular Medicine 2007, 11, 670-684.

[9] M. C. Kerr, R. D. Teasdale, Traffic 2009, 10, 364-371.

[10] M. Chabaud, M. L. Heuzé, M. Bretou, P. Vargas, P. Maiuri, P. Solanes, M. Maurin, E. Terriac, M. Le Berre, D. Lankar, T. Piolot, R. S. Adelstein, Y. Zhang, M. Sixt, J. Jacobelli, O. Bénichou, R. Voituriez, M. Piel, A.-M. Lennon-Duménil, Nature Communications 2015, 6, 7526.

[11] C. Amatore, S. Arbault, M. Guille, F. Lemaitre, Chemical Reviews 2008, 108, 2585-2621.

[12] A.-S. Cans, A. G. Ewing, J. Solid State Electrochem. 2011, 15, 1437-1450.

[13] F. Lemaitre, M. G. Collignon, C. Amatore, Electrochimica Acta 2014, 140, 457-466.

[14] R. M. Wightman, J. A. Jankowski, R. T. Kennedy, K. T. Kawagoe, T. J. Schroeder, D. J. Leszczyszyn, J. A. Near, E. J. Diliberto, O. H. Viveros, Proceedings of the National Academy of Sciences of the United States of America 1991, 88, 10754-10758.

[15] M. L. Huffman, B. J. Venton, Analyst 2009, 134, 18-24.

[16] T. J. Schroeder, J. A. Jankowski, K. T. Kawagoe, R. M. Wightman, C. Lefrou, C. Amatore, Analytical Chemistry 1992, 64, 3077-3083.

[17] P. Chen, B. Xu, N. Tokranova, X. J. Feng, J. Castracane, K. D. Gillis, Analytical Chemistry 2003, 75, 518-524.

[18] A. F. Dias, G. Dernick, V. Valero, M. G. Yong, C. D. James, H. G. Craighead, M. Lindau, Nanotechnology 2002, 13, 285-289.

[19] X. H. Chen, Y. F. Gao, M. Hossain, S. Gangopadhyay, K. D. Gillis, Lab Chip 2008, 8, 161-169.

[20] C. L. Gao, X. H. Sun, K. D. Gillis, Biomed. Microdevices 2013, $15,445-451$.

[21] X. H. Sun, K. D. Gillis, Analytical Chemistry 2006, 78, 2521-2525.
G-C. thanks "Emergences Ville de Paris 2014" Grant and Institut Universitaire de France Fellowship Program. XL thanks the China Scholarship Council for her financial support with a Ph. D. grant.

[22] A. Meunier, R. Fulcrand, F. Darchen, M. Guille Collignon, F. Lemaître, C. Amatore, Biophysical Chemistry 2012, 162, 14-21.

[23] A. Meunier, O. Jouannot, R. Fulcrand, I. Fanget, M. Bretou, E. Karatekin, S. Arbault, M. Guille, F. Darchen, F. Lemaitre, C. Amatore, Angewandte ChemieInternational Edition 2011, 50, 5081-5084.

[24] P. J. O'Connell, X. B. Wang, M. Leon-Ponte, C. Griffiths, S. C. Pingle, G. P. Ahern, Blood 2006, 107, 1010-1017.

[25] M. L. Heuze, O. Collin, E. Terriac, A.-M. LennonDumenil, M. Piel, Methods in molecular biology (Clifton, N.J.) 2011, 769, 415-434.

[26] G. Faure-Andre, P. Vargas, M.-I. Yuseff, M. Heuze, J. Diaz, D. Lankar, V. Steri, J. Manry, S. Hugues, F. Vascotto, J. Boulanger, G. Raposo, M.-R. Bono, M. Rosemblatt, M. Piel, A.-M. Lennon-Dumenil, Science 2008, 322, 1705-1710.

[27] T. Mueller, T. Duerk, B. Blumenthal, M. Grimm, S. Cicko, E. Panther, S. Sorichter, Y. Herouy, F. Di Virgilio, D. Ferrari, J. Norgauer, M. Idzko, PLoS One 2009, 4.

[28] R. Pacheco, C. E. Prado, M. J. Barrientos, S. Bernales, Journal of Neuroimmunology 2009, 216, 8-19.

[29] C. Prado, F. Contreras, H. Gonzalez, P. Diaz, D. Elgueta, M. Barrientos, A. A. Herrada, A. Lladser, S. Bernales, R. Pacheco, J. Immunol. 2012, 188, 3062-3070.

[30] K. Nakano, T. Higashi, R. Takagi, K. Hashimoto, Y. Tanaka, S. Matsushita, International Immunology 2009, 21, 645-654.

[31] S. Axelsson, R. Elofsson, B. Falck, S. Sjoborg, Acta Dermato-Venereologica 1978, 58, 31-35.

[32] A. Meunier, M. Bretou, F. Darchen, M. Guille Collignon, F. Lemaître, C. Amatore, Electrochimica Acta 2014, 126, 74-80.

[33] C. Amatore, S. Arbault, Y. Chen, C. Crozatier, F. Lemaitre, Y. Verchier, Angewandte ChemieInternational Edition 2006, 45, 4000-4003.

[34] C. Amatore, J. Delacotte, M. Guille-Collignon, F. Lemaitre, Analyst 2015, 140, 3687-3695. 